\section{Risk factors for pre-eclampsia among women at antenatal booking in Kano, Northern Nigeria}

\author{
Ibrahim A. Yakasai, ${ }^{1}$ \\ Imran O. Morhason-Bello² \\ 'Department of Obstetrics and \\ Gynaecology, Bayero University \\ Kano/Aminu Kano Teaching Hospital, \\ Kano; 'Department of Obstetrics and \\ Gynaecology, University College Hospital, \\ Ibadan, Nigeria
}

\section{Abstract}

Pre-eclampsia (PE) is an important cause of maternal mortality. There have been several studies on risk factors assessment with conflicting reports across the globe on this disease; however, rigorous recent evaluation of these factors is uncommon in this region. The aim of the present study was to determine the risks factors in the early-onset PE in Aminu Kano Teaching Hospital (AKTH), Kano (Northern Nigeria). We conducted a case-control study in Nigeria between April 2009 and January 2010 to identify the risk factors associated with the early-onset PE in women attending antenatal clinic in AKTH. Information on socio-cultural characteristics, medical history, previous obstetrics history, level of stress at home, and type of family were obtained and recorded in a proforma designed for the study. Multiple logistic regression analysis was used to determine the risk factors for PE at 95\% confidence level. Pregnant women with early-onset PE (150 in each case and control group). Risk factors associated with increased risk of early-onset PE were: history of pre-eclampsia/eclampsia (PE/E) in a previous pregnancy [adjusted odds ratio (AOR) 2.09]; exposure to passive smoking (AOR 1.34); inadequate antenatal supervision (AOR 15.21); family history of hypertension in one or more $1^{\text {st }}$-degree relative (AOR 8.92); living in a joint family (AOR 6.93); overweight (120\% to $150 \%$ of pre-pregnancy ideal body weight, AOR 4.65). Risk factors among women in Northern Nigeria are similar to those reported from other studies. Good antenatal cares, early detection, reduction of stressful conditions at home are the most important preventive measures of early-onset severe $\mathrm{PE}$ among these women.

\section{Introduction}

Pre-eclampsia (PE) is a major cause of maternal and fetal mortality and morbidity. In general, the incidence of PE ranges between 2 and $10 \%$ worldwide. ${ }^{1}$ In an average UK population, the incidence of PE is less than 1 in 20 women. ${ }^{2}$ According to the National Institute of Clinical Excellence (NICE) antenatal guidelines, a woman's level of risk for PE in a given pregnancy should be assessed at the first antenatal visit by identifying the presence of one or more predisposing historical risk factors, and they should be supervised more vigilantly and managed at centers with facilities for specialized neonatal and maternal intensive care. ${ }^{1}$ In the guidelines, some of the risk factors for the development of PE are: first pregnancy; previous $\mathrm{PE} ;>10$ years since last baby; age $>40$ years; body mass index (BMI) $>35$; family history of $\mathrm{PE}$; booking diastolic blood pressure (BP) $80 \mathrm{mmHg}$; proteinuria at booking of +1 on more than one occasion or $300 \mathrm{mg} / 24 \mathrm{~h}$; multiple pregnancy; and underlying medical conditions.

Different populations and ethno-geographical groups may have different risk factors. ${ }^{2}$ It is important to establish the individual contribution of the various risk factors for the development of PE, most relevant to the particular population. Such studies in African women are limited. The incidence of PE in Nigeria is not known. It has never been evaluated on a large randomized trial to give a true national incidence. Beside the study by Anorlu and colleagues there is none available to the best of our knowledge that attempts to estimate the incidence of PE in Lagos state. Most studies from Nigeria dealt with the incidence on eclampsia, and these vary in different geographical areas. It is as low as $0.3 \% / 100$ delivery in Calabar (Southern Nigeria) to as high as 5-9\%/100 in Kano (Northern Nigeria). Rates are generally higher in the north than the south. Several other studies have been carried out on eclampsia. ${ }^{3-5}$

In general, the diagnostic criteria for PE are hypertension (HT) and significant proteinuria. The degree of these criteria is a major determinant to the progression of the disease. Women who have had a pregnancy complicated by PE and endured significant maternal or perinatal morbidity require specific counseling regarding recurrence (range $0-5 \%$ and up to $47 \%$ ) in order to make decisions about future reproduction.

The screening tests to predict PE are also available and they are biophysical and biochemical. The most promising biophysical test is uterine artery Doppler scan. Though inexpensive in the western world, it is rarely performed in developing countries due to cost and manpower. ${ }^{6}$ Therefore, identification of clinical, laboratory and historical risk factors for the development of PE in a particular population group will guide the healthcare providers
Correspondence: Ibrahim Yakasai, Department of Obstetrics and Gynaecology, Aminu Kano Teaching Hospital, Hospital Road, PMB 3452, Kano, Nigeria.

Tel/Fax: +234.802.751.3292

E-mail: ibrahimyakasai57@hotmail.com

Key words: hypertension, pre-eclampsia, risk factors, Nigeria.

Contributions: the authors contributed equally.

Conflict of interests: the authors declare no potential conflict of interests.

Received for publication: 11 December 2012. Revision received: 9 Febraury 2013.

Accepted for publication: 16 Febraury 2013.

This work is licensed under a Creative Commons Attribution 3.0 License (by-nc 3.0).

(C)Copyright I.A. Yakasai and I. Mohassan Bello, 2013

Licensee PAGEPress, Italy

Healthcare in Low-resource Settings 2013; 1:e12 doi:10.4081/hls.2013.e12

during counseling of such women and possibly reduce the recurrence risk of PE if some modifiable risk factors (like obesity and insulinresistance) are present. The present study is proposed to identify the clinical and historical risk factors in women with early-onset PE (defined as having symptoms of PE before 34 weeks' pregnancy) ${ }^{7}$ attending a tertiary care hospital in North Nigeria, and compare these risk factors with those in women in a control group without HT.

\section{Materials and Methods}

This case-control study was conducted between April 2009 and January 2010 in the Department of Obstetrics and Gynaecology of Aminu Kano Teaching Hospital (AKTH) Kano, Nigeria. In this study PE is defined as a rise blood pressure of 140/90mmHg and $300 \mathrm{mg}$ of protein in a 24-hour urine sample in the second half of pregnancy. Severe PE is defined as a BP over $160 / 110$ with or without additional symptoms.

The study group was comprised of 150 women seen at the clinic and admitted with early-onset (34 weeks) PE with a systolic BP of $160 \mathrm{mmHg}$ or a diastolic of $110 \mathrm{mmHg}$ after 20 weeks' gestation, plus proteinuria of $2.0 \mathrm{~g} / 24 \mathrm{~h}$ (or $2+$ on qualitative examination), according to the National guidelines and the departmental protocol. ${ }^{1,8}$ The control group was comprised of 150 women admitted without HT and a BP less than 140/90 obtained on two occasions at least $6 \mathrm{~h}$ apart, for first time during pregnancy 
after 34 weeks' gestation and no proteinuria, which delivered during the same period. The control groups were normotensive women matched with the study group in terms of gestational age, parity and age. Women with uncertain gestational age, poor compliance for follow-up, whose data did not contain platelet count, renal and liver functions, were excluded. All women gave written informed consent and the study was approved by the Research Ethics Committee of the Institute. The researchers took the medical history and fill the proformas: data was collected by the principal investigator and research assistant, using a pre-tested interviewer-administered questionnaire. A detailed history, including past, personal and family history, pregnancy outcome, including delivery details and perinatal outcome and available investigations were recorded. If $\mathrm{BP}$ records in the $1^{\text {st }}$ trimester were not available, the women were examined after 12 weeks of delivery to note the presence of high BP. Though PE and HT may have different pathology, patients with existing HT were included, in order to determine those who develop superimposed PE and the role if any and influence of pre-existing HT in women who go on to develop PE. Data also exist showing that, pre-eclamptic women were more likely to have a family history of HT. H,10 $^{9}$

The stress at work was calculated by a fivelevel activity score, based on the distance and transportation from home to workplace, type and physical intensity of work and weekly working hours adapted from a study by Anorlu et al. ${ }^{11}$ The nature of work among the employed women in the present study was classified into sedentary, moderate and intense physical activity. ${ }^{12}$ Anorlu et al. had calculated the stress at home in terms of lack of home help, number of young children, stress from husband and number of extended family members living with the patient. They assess factors regarding stress at home and its role as a risk factor in PE. No particular score was given to these factors, but found stress from lack of home help to be the most important factor. The score is not validated but useful in assessing risk factors in PE among pregnant Nigerian women. We therefore chose to use it in this study.

These factors were modified to be suitable for the population enrolled in the present study and included availability of home help, resting hours and family type.

The minimum sample size was calculated to be 138 using this validated formula: ${ }^{13}$

$$
\mathrm{n}=\mathrm{z} 2 \mathrm{p}(1-\mathrm{p}) / \mathrm{d} 2
$$

where $\mathrm{n}=$ minimum sample required; $\mathrm{z}=\mathrm{stan}$ dard normal deviation $=1.96$ (at $95 \%$ confidence level); $d=$ sample error $=5 \%=0.05$; $\mathrm{p}=$ incidence of women with PE (obtained from other studies) $10 \%{ }^{1}$

$$
\begin{aligned}
\text { Therefore: } & n=(1.96) 2(0.10)(1-0.10) /(0.05) 2 \\
& n=3.84 \times 0.10 \times 0.90 / 0.0025 \\
n & =0.3456 / 0.0025 \\
& n=345 / 2.5 \\
n & =138.2
\end{aligned}
$$

To account for failed or incomplete response, the minimum sample size was increased to 150. Consecutive sampling method was used to recruit study participants

Data were analyzed using software SPSS version 11 for Windows. Analysis was done to check for range and consistency of data to determine the risk factors associated with severe PE. Odds ratio (OR) and 95\% confidence interval (CI) was calculated for each risk factor. A $P$ value $<0.05$ was considered significant.

\section{Results}

During the study period, there was a total of 2920 deliveries, of which 250 women had high diastolic BP (mild PE 38, 15.2\%; severe PE 120, 48\%; eclampsia 78, 31.2\%; chronic HT 14 , 5.6\%). Among the 150 study patients, 110 women had early-onset severe PE, 30 had eclampsia and 10 had superimposed PE on the underlying HT. All the women in the control group had normal BP at 6 weeks' postpartum.

The mean age of cases and controls were 26.03 and 26.46 years, respectively. The sociodemographic characteristics of patients are shown in Table 1. The study group had significantly more multigravid women as compared with the control group [crude odds ratio (COR) 1.92; 95\% CI 1.05-3.52]. The socio-economic status was categorized into five classes according to Olusanya's classification. ${ }^{14}$ In the study group, $81 \%$ of women belong to the lower and lower-middle classes as compared with only $43 \%$ in the control group, which is statistically significant.

Table 2 shows the medical and obstetric characteristics of patients. In the present study, the mean gestation at which women developed severe PE/E was 34 weeks, while none among the control developed it. The perinatal mortality in the study group was $42 \%$ as compared with none in the control group. The study group women had severe disease, as 70\% had BP 4180/120; 68\% had urine albumin 3+; $35 \%$ had HELLP syndrome; $17 \%$ had deranged renal functions and $25 \%$ had eclampsia.

The frequencies of antenatal visits are categorized into adequate, intermediate and inadequate, according to Kessner index criteria. ${ }^{15}$ The mean weight of cases and controls were $68.03+5.61 \mathrm{~kg}$ (range 50-81 kg) and 66.58 + $8.48 \mathrm{~kg}$ (range $44-95 \mathrm{~kg}$ ), respectively. Since there are no nomograms of BMI during pregnancy, and the pre-pregnancy weight is not always recorded, these women were categorized into four groups on the basis of the current pregnancy weight expressed as a percentage of the pre-pregnancy ideal body weight. ${ }^{16}$ Two women in the study group had gestational diabetes mellitus (GDM) controlled on diet and four had frank diabetes (type 1).

A history of previous PE/E was associated with more than 18 times increased risk of developing PE (COR 18.86; 95\% CI 2.55389.25). As smoking has been found to be a factor that reduces the risk of $\mathrm{PE} / \mathrm{E}$, we considered exposure to passive smoking to represent this factor, since none of the participants in the study group gave a history of smoking. Exposure to passive smoking is defined as the involuntary exposure to a combined but diluted cigarette side-stream smoke and the exhaled smoke from the smokers. ${ }^{17}$

The level of stress at home and at work is shown in Table 3. Resting hours were defined as the availability of resting time for $2 \mathrm{~h}$ in the day and $7 \mathrm{~h}$ at night. A monogamous family consists of the married couple and their dependent children occupying the same dwelling place. The polygamous consists of a number of wives married to one man living with their children; other members of the family related may also be living in the same compound.

\begin{tabular}{|c|c|c|c|c|c|c|c|}
\hline \multirow[t]{2}{*}{ Variables } & \multicolumn{2}{|c|}{ Cases $(n=150)$} & \multicolumn{2}{|c|}{ Controls $(n=150)$} & \multirow[t]{2}{*}{ OR } & \multirow[t]{2}{*}{$95 \%$ CI } & \multirow[t]{2}{*}{ P value } \\
\hline & n & $\%$ & $\mathrm{n}$ & $\%$ & & & \\
\hline \multicolumn{8}{|l|}{ Age (years) } \\
\hline$<20$ & 10 & 6.67 & 14 & 9.33 & 0.69 & $0.28-1.73$ & 0.5 \\
\hline $20-34$ & 122 & 81.33 & 126 & 84 & 0.83 & $0.44-1.57$ & 0.65 \\
\hline$\geq 35$ & 18 & 12 & 10 & 6.67 & 1.91 & $0.80-4.63$ & 0.16 \\
\hline \multicolumn{8}{|c|}{ Gravidity } \\
\hline Primigravidae & 66 & 44 & 75 & 50 & 0.6 & $0.37-0.97$ & 0.04 \\
\hline Multigravidae & 84 & 56 & 75 & 50 & 1.27 & $0.79-2.06$ & 0.35 \\
\hline
\end{tabular}

Pre-pregnancy HT was taken as a significant risk factor for the development of early-onset severe $\mathrm{PE}$ on the basis of a highly significant $\mathrm{P}$ value $(\mathrm{P}<0.0003)$.

Table 1. Demographic data of women in study and control groups.

OR, odds ratio; $\mathrm{CI}$, confidence interval. 


\section{Discussion}

In this study the finding revealed that a history of PE, pre-existing diabetes, multiple pregnancies, and family history, a raised BMI greater than 35 at booking, maternal age greater than 35 years, underlying renal disease, HT, more than 5 years since last pregnancy, and raised BP at booking all increased the risk of women developing PE.
The study further shows that exposure to passive smoking is a significant risk factor for the early-onset severe PE. However, other studies have reported that cigarette smoking during pregnancy was associated with a $32 \%$ and $35 \%$ reduction in the risk of $\mathrm{PE}$.,18 The biologic mechanism by which cigarette smoking during pregnancy may reduce the risk of $\mathrm{PE}$ is not clear. It may be possible that smoking leads to the earlier termination of pregnancies - miscarriage, preterm delivery, or abruption -

Table 2. Medical and obstetric characteristics of women in study and control groups.

\begin{tabular}{|c|c|c|c|c|c|}
\hline Variables & Cases (n) & Controls (n) & OR & $95 \% \mathrm{CI}$ & P value \\
\hline \multicolumn{6}{|l|}{ Kessner index criteria } \\
\hline Adequate & 28 & 101 & 0.11 & $0.06-0.2$ & $<0.0001$ \\
\hline Intermediate & 43 & 49 & 0.83 & $0.49-4.28$ & 0.5 \\
\hline Inadequate & 69 & 44 & 2.05 & $1.24-3.4$ & 0.004 \\
\hline \multicolumn{6}{|l|}{ Antenatal complications } \\
\hline Multiple pregnancy (twins) & 12 & 8 & 1.54 & $0.57-4.28$ & - \\
\hline Gestational diabetes & 9 & 20 & 0.41 & $0.17-1.00$ & - \\
\hline Abruptio placenta & 25 & 0 & - & - & - \\
\hline Placenta praevia & 4 & 0 & - & - & - \\
\hline PROM & 7 & 4 & 1.79 & $0.46-7.44$ & - \\
\hline \multicolumn{6}{|c|}{ BMI/Pregnancy weight categories } \\
\hline Normal weight $(80-120 \%)$ & 39 & 76 & 0.34 & $0.2-0.57$ & $<0.0001$ \\
\hline Overweight (>120-150\%) & 105 & 66 & 0.92 & $0.6-1.42$ & 0.77 \\
\hline Obese $(>150 \%)$ & 6 & 8 & 0.74 & $0.22-2.42$ & 0.78 \\
\hline \multicolumn{6}{|l|}{ Medical disorders } \\
\hline Pre-pregnancy HT & 24 & 0 & - & & $<0.0001$ \\
\hline Diabetes mellitus & 4 & 0 & - & - & 0.06 \\
\hline Renal disease & 5 & 0 & - & - & 0.03 \\
\hline Hypothyroidism & 2 & 0 & 2.01 & $0.14-56.69$ & 0.5 \\
\hline \multicolumn{6}{|l|}{ Previous obstetric outcome } \\
\hline Abortions & 38 & 40 & 0.93 & $0.54-1.61$ & 0.9 \\
\hline $\mathrm{PE} / \mathrm{E}$ & 32 & 2 & 20.1 & $4.56-123.7$ & $<0.0001$ \\
\hline Gestational HT & 12 & 3 & 4.26 & $1.09-19.45$ & 0.03 \\
\hline \multicolumn{6}{|c|}{ Family history of medical disorders } \\
\hline $\mathrm{HT}$ & 84 & 36 & 4.03 & $2.39-6.82$ & $<0.0001$ \\
\hline Diabetes mellitus & 38 & 42 & 0.87 & $0.5-1.50$ & 0.7 \\
\hline $\mathrm{PE} / \mathrm{E}$ & 12 & 6 & 2.09 & $0.7-6.44$ & 0.22 \\
\hline \multicolumn{6}{|l|}{ Smoking } \\
\hline YES & 4 & 3 & 1.34 & $0.25-7.7$ & 0.5 \\
\hline NO & 146 & 147 & - & - & - \\
\hline
\end{tabular}

OR, odds ratio; $\mathrm{CI}$, confidence interval; Kessner index criteria, adequacy of prenatal care; PROM, prelabor rupture of membranes; BMI/Pregnancy weight categories, current weight expressed as a percentage of ideal pre-pregnancy weight; BMI, body-mass index; HT, hypertension; PE/E, pre-elcampsia/eclampsia.

Table 3. Level of stress at home and at work of women in study and control groups.

\begin{tabular}{lccccc}
\hline Variables & Cases (n) & Controls (n) & OR & $95 \%$ CI & P value \\
$\begin{array}{l}\text { Employment during pregnancy } \\
\quad \text { YES }\end{array}$ & 25 & 34 & 0.68 & $0.37-1.26$ & 0.25 \\
$\quad$ NO & 125 & 116 & - & - & - \\
$\begin{array}{l}\text { Availability of home help } \\
\text { (housemaid/relations) }\end{array}$ & & & & & \\
$\quad$ YES & 60 & 111 & 0.23 & $0.14-0.39$ & $<0.0001$ \\
$\quad$ NO & 90 & 39 & - & - & - \\
\hline $\begin{array}{l}\text { Family type } \\
\quad \text { Monogamous }\end{array}$ & 68 & 67 & 1.03 & $0.64-1.66$ & 0.1 \\
$\quad$ Polygamous & 82 & 83 & 0.97 & $0.6-1.57$ & 0.1 \\
\hline OR, odds ratio; Cl, confidence interval. & & & & &
\end{tabular}

which would otherwise be destined to manifest as PE. ${ }^{17}$

Family history of PE was reported to be a risk factor and nearly triples the risk for PE. ${ }^{17}$ However, in another study, it did not emerge as a significant risk factor. ${ }^{19}$ History of pre-pregnancy HT was a significant risk factor associated with early-onset severe PE in univariate analysis (RR 2.14), which is in agreement with other studies. Brown and colleagues ${ }^{20}$ found that the prevalence of chronic HT was higher in women who develop PE than women who do not $(12.1 \%$ vs $0.3 \%)$.

The results of the present study regarding the stress-related factors shows that women of lower socio-economic status and living in a joint family have an increased risk of severe early-onset PE. It is likely that these women may have increased household work due to more family members in a joint family leading to increased physical and mental stress, which may predispose them to develop severe earlyonset PE.

Chronic autoimmune disease like hypothyroidism in this study remained a significant risk factor in the development of PE. In a matched case-controlled study Wolfberg et al. ${ }^{21}$ found that women who develop PE were more likely to have an autoimmune disease. In the present study, of the 150 women with severe PE, 4/8 women with hypothyroidism had chronic HT as compared to 26/92 euthyroid women (50 vs 28\%). The final verdict as to whether hypothyroidism per se is a significant risk factor for PE or whether it is a contributory factor due to its association with chronic HT will be clarified by larger studies.

Risk factors may be specific to the mother or the pregnancy. Some such as primigravidity or past history of PE are well known. Primigravidae are 15 times more likely to develop protienuric PE than parous women, ${ }^{21}$ which is similar to the present study where nulliparity almost triples the risk for PE. However, Sibai et al. ${ }^{22}$ showed that HDP are more frequent in multigravidae suggesting that multigravidae are more likely to have early-onset and severe disease.

In the present study, only a few women were employed during pregnancy in both the study (6\%) and the control (15\%) groups; and being unemployed appeared to be a risk factor for early-onset severe PE in univariate analysis (COR 2.76). On the other hand, some authors have reported that employment during pregnancy is a significant risk factor for developing PE. ${ }^{12,23}$ This difference may be due to a lesser number of employed women in both groups. The present population of Northern Nigeria women represents a group, among which most women are not formally employed, but may be working much harder in large joint families, which was a significant risk factor for severe early-onset PE (AOR 6.93). 
There have been conflicting reports on the impact of maternal age on PE. We did not find age to be a significant risk factor, which is consistent with a similar study by Eskenazi $e t$ al. who conducted their study in North California (USA) among black pregnant women attending antenatal clinic. ${ }^{24}$ However, a Nationwide US data suggest that the risk of PE increases by $30 \%$ for every additional year of age after 34 years. ${ }^{25}$ This may be probably due to the fact that the majority of our study participants concluded their childbearing by the age of 30 . Furthermore, multiple pregnancy as a significant risk factor was also observed in this study. Several studies have shown that when a woman is pregnant with twins her risk of $\mathrm{PE}$ nearly triples, neither the chronicity nor zygosity alter this increased risk. ${ }^{11,26,27}$

In summary, factors which emerged to be significant in the multivariate analysis were: history of $\mathrm{PE} / \mathrm{E}$ in a previous pregnancy (AOR 71.40); exposure to passive smoking (AOR 16.40); inadequate antenatal supervision (AOR 15.21); family history of HT in one or more $1^{\text {st }-d e g r e e ~ r e l a t i v e s ~(A O R ~ 8.92) ; ~ l i v i n g ~ i n ~}$ a joint family (AOR 6.93); overweight (4120$150 \%$ of pre-pregnancy ideal body weight, AOR 4.65) and lower socio-economic class (Olusanya's class III-V) (AOR 3.00). The results of the present study are in agreement with other studies. ${ }^{10,19,24,28}$

A history of previous PE/E was associated with more than 18 times increased risk of developing $\mathrm{PE}$, while pre-pregnancy ideal body weight found to be least associated with PE.

\section{Conclusions}

Based on the presence of risk factors identified in the present study, women may be counseled prior to or during pregnancy and advised to have adequate antenatal supervision in a hospital with appropriate facilities for caring of women with severe PE and pre-term neonates. This may help to constitute a risk model as practice in other units, thereby reducing the attendant maternal and perinatal complications.

\section{References}

1. National Institute for Health and Care
Excellence. NICE Guidelines CG6 Antenatal care-routine care for the healthy pregnant woman. London: NICE Publ.; 2003.

2. Milne F, Redman C, Walker J, et al. The pre-eclampsia community guideline (PRECOG): how to screen for and detect onset of pre-eclampsia in the community. Brit Med J 2005;330:576-80.

3. Adamu YM, Salihu HM, Sarthiakumar N, Alexandra R. Maternal mortality in Northern Nigeria: a population based study. Eur J Obstet Gyn R B 2003;109:1539.

4. Yakasai IA, Gaya SA. Maternal and fetal outcome in patients with eclampsia at Murtala Muhammad Specialist hospital Kano, Nigeria. Ann Afr Med 2011;10:305-9.

5. Itam IH. Sociodemographic determinants of Eclampsia in Calabar: a 10 year review. Mery Slessor. J Med 2003;3:72-4.

6. Lawoyin T0, Ani F. Epidemiological aspect of pre-eclampsia in Saudi Arabia. E Afr Med J 1996;73:404-6.

7. Oettle C, Hall D, Roux A, Grove D. Early onset severe preeclampsia: expectant management at a secondary hospital in close association with a tertiary institution. Brit J Obstet Gynaec 2005;112:84-8.

8. Duckitt K, Harrington D. Risk factors for pre-eclampsia at antenatal booking: systematic review of controlled studies. Brit Med J 2005;2:1-7.

9. Conde-Agudelo A, Belizan JM. Risk factors for pre-eclampsia in a large cohort of Latin American and Caribbean women. Brit J Obstet Gynaec 2000;107:75-83.

10. Cincotta RB, Brennecke SP. Family history of pre-eclampsia as a predictor for preeclampsia in primigravidas. Int $\mathrm{J}$ Gynecol Obstet 1998;60:23-7.

11. Anorlu RI, Iwuala NC, Odum CU. Risk factors for preeclampsia in Lagos, Nigeria. Aust NZ J Obstet Gyn 2005;45:278-82.

12. Spinillo A, Capuzzo E, Colonna L, Piazzi G, Nicola S, Baltaro F. The effect of work activity in pregnancy on the risk of severe pre-eclampsia. Aust NZ J Obstet Gyn 1995;35:380-5.

13. Lwanga SK, Lemeshow S. Sample size determination in health studies: a practical manual. Geneva: World Health Organization ed.; 1991.

14. Olusanya 0, Okpere E, Ezimokhai M. The importance of social class in voluntary fertility control in developing country. West Afr J Med 1985;4:205-12.
15. Kessner D. Infant death: an analysis by maternal risk and health care. 1 . Washington, DC: National Academy of Sciences ed.; 1973.

16. DeVader SR, Neeley HL, Myles TD, Leet TL. Evaluation of gestational weight gain guidelines for women with normal prepregnancy body mass index. Obstet Gynecol 2007;110:745-51.

17. Salafia C, Shiverick K. Cigarette smoking and pregnancy II: vascular effects. Placenta 1999;20:273-9.

18. Mostello D, Kallogjeri D, Tungsiripat R, Leet T. Recurrence of preeclampsia: effects of gestational age at delivery of the first pregnancy, body mass index, paternity, and interval between births. Am J Obstet Gynecol 2008;199:1-7.

19. Nanjundan P, Bagga R, Kalra JK, et al. Risk factors for early onset pre-eclampsia and eclampsia among north Indian women. J Obstet Gynaecol 2011;31:384-9.

20. Brown MA, Davis GK, McHugh L. The prevalence and clinical significance of nocturnal hypertension in pregnancy. $\mathrm{J}$ Hypertens 2001;19:1437-44.

21. Wolfberg AJ, Lee-Parritz A, Peller AJ, Lieberman ES. Obstetric and neonatal outcomes associated with maternal hypothyroid disease. J Matern-Fetal Neo M 2005; 17:35-8.

22. Sibai BM, Gordon T, Thom E, et al. Risk factors for pre-eclampsia in a healthy parous women: a prospective multicenter study. Am J Obstet Gynecol 1995;172:642-8.

23. Lee CJ, Hsieh TT, Chiu TH, et al. Risk factors for pre-eclampsia in an Asian population. Int J Gynecol Obstet 2000;70:327-33.

24. Eskenazi B, Fenster L, Sidney S. A multivariate analysis of risk factors for preeclampsia. J Amer Med Assoc 1991;266: 237-41.

25. Redman CWG. Hypertension. In: de Sweit M, ed. Medical disorders in obstetrics practice. 4th ed. Oxford: Blackwell Scientific Publications; 2002. pp 159-197.

26. Campbell DM, MacGillivray I. Preeclampsia in twin pregnancies: incidence and outcome. Hypertens Pregnancy 1999;18: 197-207.

27. Stone JL, Lockwood CJ, Berkowitz GS, et al. Risk factors for severe pre-eclampsia. Obstet Gynecol 1994;83:357-81.

28. Odegard RA, Vatten LJ, Nielsen ST, et al. Risk factors and clinical manifestation of pre-eclampsia. Brit J Obstet Gynaec 2000;107:1410-6. 